EDITORIAL

\title{
Implantable cardioverter-defibrillators for children and young adolescents: mortality benefit confirmed-what's next?
}

\section{S F Sears, J B Conti}

The implantable cardioverter-defibrillator can present significant psychological difficulties for some younger patients, not least because of increased lifestyle disruption and the likely experience of shock

See end of article for authors' affiliations

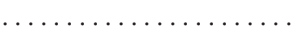

Correspondence to: Samuel F Sears, PhD, University of Florida, Department of Clinical \& Health Psychology, Box 100165 UF Health Science Center, Gainesville, FL 32610, USA; ssears@hp.ufl.edu
$\mathrm{T}$ he implantable cardioverter-defibrillator (ICD) is the treatment of choice for potentially life threatening ventricular arrhythmias. Consistent with the adult clinical trial data for both primary and secondary prevention, Gradaus and colleagues ${ }^{1}$ followed children and young adolescents with ICDs for an average of four years and found that the children's total survival was excellent (95.8\%). The demonstrated mortality benefit demonstrated by Gradaus and colleagues $^{1}$ in this issue of Heart allows us to turn our attention to psychosocial issues and quality of life (QoL) of young ICD patients. ${ }^{2}$

We reported that younger age and greater frequency of ICD firings were the two most commonly reported ICD specific risk factors for psychological distress. ${ }^{3}$ Younger patients (50 years and younger) may experience greater problems because of increased lifestyle disruption and distressing social comparisons. ${ }^{2}$ In addition to adjusting to the risk of potentially life threatening arrhythmias, young patients must deal with the presence of the ICD device, the likely experience of life saving shock, and the social and lifestyle ramifications of the ICD. Not surprisingly, the ICD can present significant psychological difficulties for some young patients.

In adults, the occurrence of ICD specific fears and symptoms of anxiety (for example, excessive worry, physiological arousal) are the most common psychological symptoms experienced by ICD recipients, with approximately $13-38 \%$ of recipients experiencing diagnosable levels of anxiety. Depressive symptoms are reported at rates that are generally consistent with other cardiac populations (24-33\%). ${ }^{3}$ A national US sample of healthcare providers estimated that $10-20 \%$ of their ICD patients experience reductions in QoL, emotional wellbeing, and family relationships. ${ }^{4}$ We would expect to see the same magnitude of distress, if not greater, in children.

\section{QUALITY OF LIFE IMPACT}

ICD shock is clearly the primary culprit when patients describe a decrease in quality of life, and coping with both inappropriate and appropriate
Heart 2004;90:241-242. doi: 10.1136/hrt.2003.021295

shocks remains the most significant psychosocial challenge for ICD populations. ${ }^{5}$ Schron and

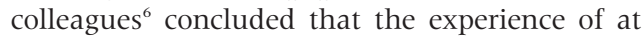
least one ICD shock was associated with reduced mental wellbeing and physical functioning. Irvine and associates ${ }^{7}$ demonstrated that five or more shocks was the threshold for a decreased quality of life outcome. Although ICD shocks feel the same whether they are appropriate or inappropriate, coping with inappropriate shocks is particularly difficult and may be associated with increased distress ${ }^{8}$ and potential distrust of the accuracy and effectiveness of the ICD. The potential differential response of patients to inappropriate versus appropriate shocks underscores the importance of psychological factors in the adjustment to the ICD.

The success described by Gradaus and colleagues ${ }^{1}$ is tempered by the percentage of inappropriate shocks $(38 \%)$ that children appeared to encounter. Although inappropriate treatment in adult ICD patients has dramatically fallen over the last several years, inappropriate therapy in children remains a problem. Data from Chechin and colleagues ${ }^{9}$ indicated that approximately $24 \%$ of children with ICDs eventually encounter lead fracture associated with physical growth, an obvious precursor to inappropriate shock. Taken together with other recent paediatric reports, ${ }^{10}$ the available studies suggest that the ICD for young children and young adults provides mortality benefit, but children may experience a disproportionate number of lead problems and inappropriate shocks. Although this should not prevent implantation of devices in those patients in whom they are clearly life saving, these data should be considered by the physician, patient, and families involved before implantation, particularly for primary prevention.

\section{ICD TREATMENT VERSUS}

\section{ANTIARRHYTHMIC MEDICATION}

QoL research in adults shows that ICD treatment is at least equal to or better than antiarrhythmic medications on patient reported and objective indicators of QoL. ${ }^{11-13}$ For example, a large randomised controlled trial (Canadian implantable defibrillator study) comparing antiarrhythmic medications versus ICDs indicated that QoL was significantly better for ICD patients in all spheres, except for pain and social functioning. ${ }^{7}$ Unfortunately, data regarding QoL in young ICD patients does not exist.

Based on the information available, routine psychological care for all ICD patients is 
reasonable, ${ }^{14}$ and young ICD patients are a subgroup well suited for this care. Specifically, patient adjustment and peer acceptance of the ICD are key developmental milestones for the young ICD patient. Descriptive and prospective research designs are still needed to address the substantial psychosocial changes and specific lifestyle and activity recommendations for young patients with ICDs. Restriction of normal activity may be particularly heinous in the young patient. Although to do no harm is the first medical objective, prohibition of desired physical activity is also problematic. In summary, we need data describing how young ICD patients perform when they do return to normal life. Returning to a full life is the hallmark feature of quality of life. Comprehensive, interdisciplinary care plans, such as those reported by Fitchet and colleagues, ${ }^{15}$ demonstrate that an exercise and stress management programme is a safe and valuable addition for ICD patients and results in reduced anxiety and improved exercise capacity. The current study by Gradaus and colleagues ${ }^{1}$ confirms mortality benefits, which if coupled with increased attention to QoL, will ultimately provide ideal health outcomes for young ICD patients.

\section{Authors' affiliations}

S F Sears, Department of Clinical Health Psychology, University of Florida, Gainesville, Florida, USA

J B Conti, Division of Cardiovascular Medicine, University of Florida

\section{REFERENCES}

1 Gradaus R, Wollmann C, Köbe J, et al. Potential benefit from implantable cardioverter-defibrillator therapy in children and young adolescents. Heart 2004:90:328-9.
2 Sears SF, Burns J, Handberg E, et al. Young at heart: understanding the unique psychosocial adjustment of young ICD patients. PACE 2001;24:1113-7

3 Sears SF, Todaro JF, Saia TL, et al. Examining the psychosocial impact of implantable cardioverter defibrillators: a literature review. Clin Cardiol 1999;22:481-9.

4 Sears SF, Todaro JF, Urizar G, et al. Assessing the psychosocial impact of the ICD: a national survey of implantable cardioverter defibrillator health care providers. PACE 2000;23:939-45.

5 Sears SF, Conti JB. Understanding ICD shocks and storms: medical and psychosocial considerations for research and clinical care. Clin Cardiol 2003;26:107-11

6 Schron EB, Exner DV, Yao Q, et al. Quality of life in the antiarrhythmics versus implantable defibrillators trial: impact of therapy and influence of adverse symptoms and defibrillator shocks. Circulation 2002;105:589-94.

7 Irvine J, Dorian P, Baker B, et al. Quality of life in the Canadian implantable defibrillator study (CIDS). Am Heart J 2002;144:282-9.

8 Burgess ES, Quigley JF, Moran G, et al. Predictors of psychosocial adjustment in patients with implantable cardioverter defibrillators. PACE 1997;20:1790-5

9 Chechin F, Alexander ME, Walsh EP, et al. Pattern of initiation of ventricular arrhythmias in pediatric and congenital heart disease patients with implantable defibrillators. PACE 2003;26:1061.

10 Stefanelli CB, Bradley DJ, Leroy S, et al. Implantable cardioverter defibrillator therapy for life-theratening arrhythmias in young patients. J Intervent Cardiac Electrophys 2002:6:235-44.

11 Burke JL, Hallas CN, Clark-Carter D, et al. The psychosocial impact of the implantable cardioverter defibrillator: a meta-analytic review. $\mathrm{Br} J$ Health Psychol 2003;8:165-78

12 Herbst JH, Goodman M, Feldstein S, et al. Health related quality of life assessment of patients with life-threatening ventricular arrhythmias. PACE 1999;22:915-26.

13 Sears SF, Conti JB. Current views on the quality of life and psychological functioning of implantable cardioverter defibrillator patients. Heart 2002;87:488-93

14 Sotile WM, Sears SF. You can make a difference: brief psychosocial interventions for ICD patients and their families. Minneapolis: Medtronic, 1999

15 Fitchet A, Doherty PJ, Bundy C, et al. Comprehensive cardiac rehabilitation programme for implantable cardioverter defibrillator patients: a randomized controlled trial. Heart 2003;89:155-60.

\section{IMAGES IN CARDIOLOGY}

\section{Acute incomplete thrombotic occlusion of distal left main coronary artery treated by tissue plasminogen activator}
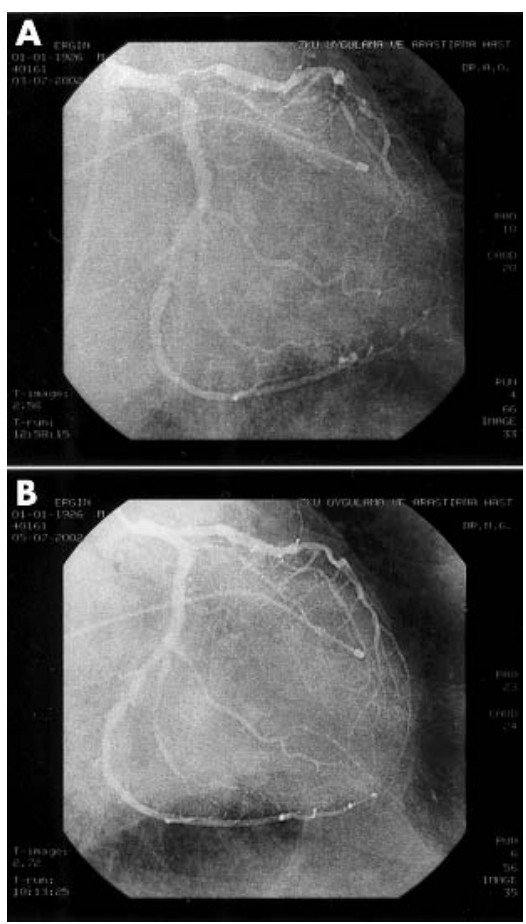

A 76 year old man presented with chest pain; he had a personal history of hypertension diabetes mellitus, and pneumoconiosis. He was hospitalised because of an inferior myocardial infarction. On the fifth day of his clinical follow up in the coronary intensive care unit, the patient experienced chest discomfort and palpitation, and an ECG revealed atrial fibrillation and ST segment depression. Afterwards, coronary angiographic examination revealed a thrombus that was narrowing the lumen to $80 \%$ at the bifurcation of the left anterior descending coronary artery (LAD) and the left circumflex coronary artery (LCx) distal to the left main coronary artery (panel A). TIMI II flow was present at the distal LAD and LCX. The patient was treated with tissue plasminogen activator (tPA) because of intracoronary thrombus. One hour after the onset of treatment, the angina pectoris ceased and a normal sinus rhythm returned. ST segment depressions and $\mathrm{T}$ wave inversions on anterior derivatives returned to normal. Cardiac troponin I concentration increased to $2 \mathrm{ng} / \mathrm{dl}$ (normal limits $0.01-$ $0.1 \mathrm{ng} / \mathrm{dl}$ ). The patient was diagnosed with non-ST elevation myocardial infarction. Coronary angiography was repeated two days later. The large thrombus in the distal left mean coronary artery was found to have resolved (panel B).

Several pathogenic processes, besides atherosclerosis, are known to involve the coronary arteries and to be responsible for severe acute coronary syndromes. Coronary embolism is included among non-atherosclerotic entities causing acute myocardial infarction and should be suspected in the presence of atrial fibrillation, and left atrial or ventricular thrombus. We report a case of distal left main coronary artery thrombus which was detected by coronary angiography and treated by tissue plasminogen activator. 